\title{
Design Study of a Two-Cavity Monotron
}

\author{
Joaquim J. Barroso \\ National Institute for Space Research-INPE \\ 12227-010 S. José dos Campos, SP, Brazil
}

\begin{abstract}
In its classical embodiment, the monotron has its conversion efficiency limited to $20.0 \%$. But we demonstrate here that on considering a stepped electricfield axial profile the monotron efficiency can attain the theoretical maximum value of $57.0 \%$, almost three times as high as that obtained from the conventional uniform field distribution. This is accomplished by using two cavities electromagnetically coupled by an annular slit crossed by an electron stream. From a one-dimensional analysis, a two-cavity monotron is developed to operate in the $\mathrm{TM}_{010}$ mode at $4.0 \mathrm{GHz}$ when self-excited by a 20 $\mathrm{keV}, 10 \mathrm{~A}$ hollow electron beam. The device operation is examined through a 21/2-D particle-in-cell (PIC) simulation giving a $47.5 \%$ conversion efficiency. Departure from the theoretically predicted efficiency is explained by beam-thickness effects.
\end{abstract}

Keywords: microwave generation; transit-time tubes

\section{Introduction}

The present paper demonstrates that the monotron [1-4] interaction can achieve a conversion efficiency as high as $57.0 \%$ by considering a stepped axial electric-field profile along the electron stream. This is accomplished by using two cavities electromagnetically coupled by an annular slit. Both cavities are carefully tailored so that a smaller cavity premodulates the beam while a second and larger resonant cavity provides the main interaction space. Such axial electric field profiles are analytically described by $\tanh (\mathrm{z})$ functions. A detailed calculation example considers a $10 \mathrm{~A}, 20 \mathrm{keV}$ hollow electron beam driving a 4.0 $\mathrm{GHz}, \mathrm{TM}_{010}$-mode cavity, whose operation is examined through a particle-in-cell (PIC) simulation which indicates a conversion efficiency of $47.0 \%$ inferred from the energy distribution of the beam electrons collected in the downstream end of the cavity.

Synthesis of a stepped electric profileFirst, we consider stepped profiles analytically described by $f(\widetilde{z})=A_{1}+A_{2} \tanh \left[A_{3}\left(\widetilde{z}-A_{4}\right)\right] \quad$ with $A_{1}+A_{2}=1.0$, $A_{3} \approx 5.0, \quad A_{4} \approx 1.0 \quad$ such that $f(\widetilde{z} \gg 1) \rightarrow 1$, $f(\widetilde{z}<<1) \rightarrow A_{1}-A_{2}$. Using the numerical treatment described in [3] to calculate the corresponding conversion efficiencies, we arrive at the results given in Tab. 1. At injection energies $\mathrm{W}_{0}=10,20$ and $50 \mathrm{keV}$, we see that the resulting conversion efficiency reaches up $57.0 \%$. The next issue is to find a practical realization of such field distributions. To this end, we shall synthesize a $4.0 \mathrm{GHz}$, $\mathrm{TM}_{010}$ monotron on the basis of the field profile with
$\mathrm{A}_{3}=6.0$ in Tab. 1; this profile is illustrated in Fig. 1. The total cavity length $d$ is determined through $d(\mathrm{~cm})=(15 / \pi) \tilde{d} / f(\mathrm{GHz})$, which gives $d=3.8 \mathrm{~cm}$ for $f=4.0 \mathrm{GHz}$ and $\tilde{d}=3.16$. The length of the prebunch cavity is determined by $\mathrm{A}_{4}=1.1$ (the position that corresponds to the middle of the transition from the lower level $\mathrm{A}_{1}-\mathrm{A}_{2}$ to the upper normalized level $\mathrm{A}_{1}+\mathrm{A}_{2}=1$ ), which being denormalized gives $d_{l}=1.3 \mathrm{~cm}$. The radius of the larger cavity is jointly set by the chosen operating mode $\left(\mathrm{TM}_{010}\right)$ and frequency $(4.0 \mathrm{GHz})$ through $R_{2}(\mathrm{~cm})=(15 / \pi) \chi_{01} / f(\mathrm{GHz})$ giving $R_{2}=2.9 \mathrm{~cm}$, where $\chi_{01}=2.4048$. Then the radius $R_{1}$ of the first cavity is made smaller than $R_{2}$; next, both radii and the slit width are further adjusted until the upper and lower levels of the resulting transition approaches the prescribed ratio 1.0/0.32 (Fig. 1). This procedure has been carried out by numerically simulating the electromagnetic structure, yielding a final with $\mathrm{R}_{1}=2.6 \mathrm{~cm}, \mathrm{R}_{2}=2.8 \mathrm{~cm}$ and a $0.1-\mathrm{cm}$ thick annular slit.

Table 1. Maximum conversion efficiencies for some stepped field profiles along with input energy, optimum electric field amplitude and cavity length.

\begin{tabular}{|c|c|c|c|c|}
\hline$W_{0}$ & $f(z)$ & $\widetilde{E}_{0}$ & $\widetilde{d}$ & $\eta(\%)$ \\
\hline \hline 10 & $0.636+0.363 \tanh [3.6(\mathrm{z}-0.8)]$ & 0.11 & 2.24 & 56.8 \\
\hline 20 & $0.636+0.363 \tanh [3.6(\mathrm{z}-1.1)]$ & 0.16 & 3.10 & 57.3 \\
\hline 20 & $0.660+0.340 \tanh [6.0(\mathrm{z}-.1)]$ & 0.15 & 3.16 & 56.5 \\
\hline
\end{tabular}

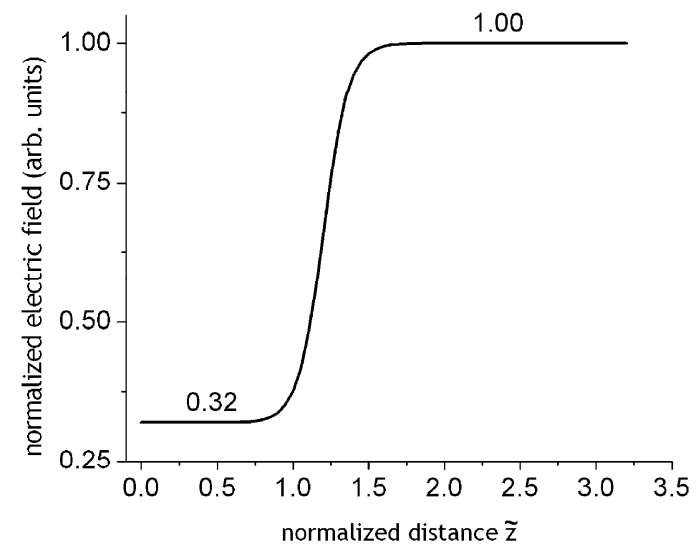

Figure 1. Stepped profile described by the analytical function $f(z)=0.66+0.34 \tanh [6.0(z-1.1)]$. 


\section{Simulation of the two-cavity monotron}

Using the particle-in-cell code KARAT [5], a 21/2-D electromagnetic simulation is performed to verify onedimensional predictions and also to ascertain the electronic efficiency of beam-to-microwave power conversion in the two-cavity monotron. Immersed in a $1.0-\mathrm{kG}$ magnetic field to ensure rectilinear flow, a 20 $\mathrm{keV}, 10 \mathrm{~A}$ hollow beam is then injected from the left into the cavity. Symmetric about the z-axis, the beam is $0.2 \mathrm{~cm}$ thick with a mean radius of $0.8 \mathrm{~cm}$, as displayed in Figs. 6(a)-6(c). This sequence of plots shows the beam configuration at (a) earlier times, (b) at the time the efficiency
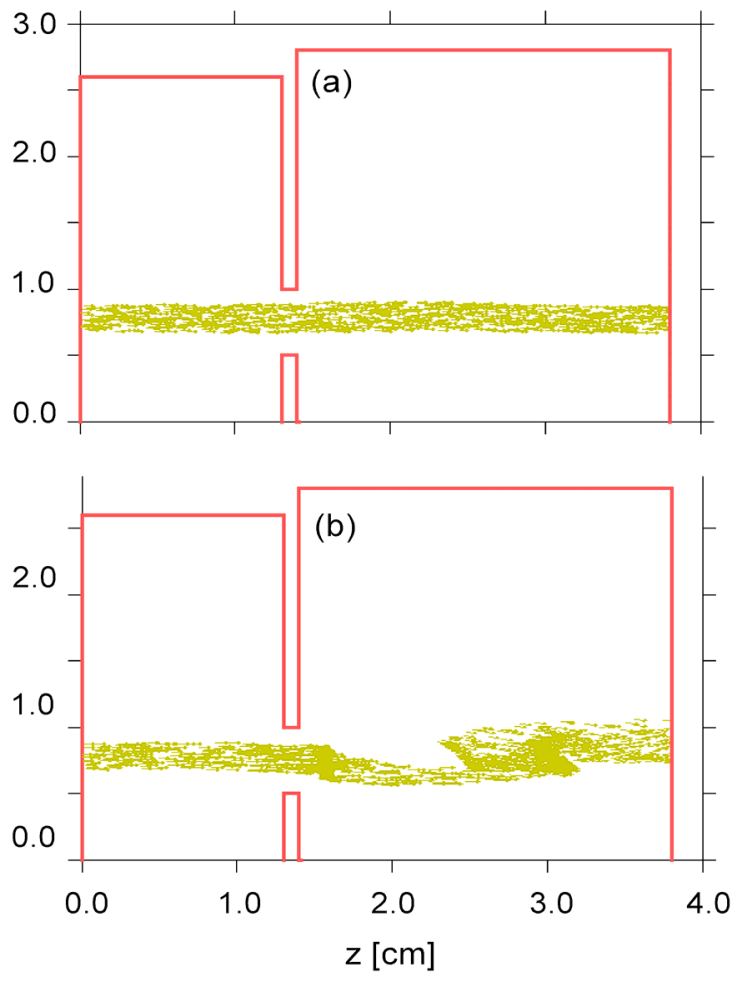

Figure 2. Space configuration of the hollow electron beam confined by a $1.0-\mathrm{kG}$ magnetic field (a) at earlier times (10 ns) and (b) in the saturation regime (>750 ns).

tops its maximum, and (c) in the saturation regime. Under this condition the conversion efficiency reaches the value of $47.5 \%$, as indicated by the kinetic-energy distribution (Fig. 7) of beam electrons collected at the end section, $\mathrm{z}=3.8 \mathrm{~cm}$, of the cavity. This optimal efficiency corresponds to the average power of $95.0 \mathrm{~kW}$ delivered by the beam to the cavity field.

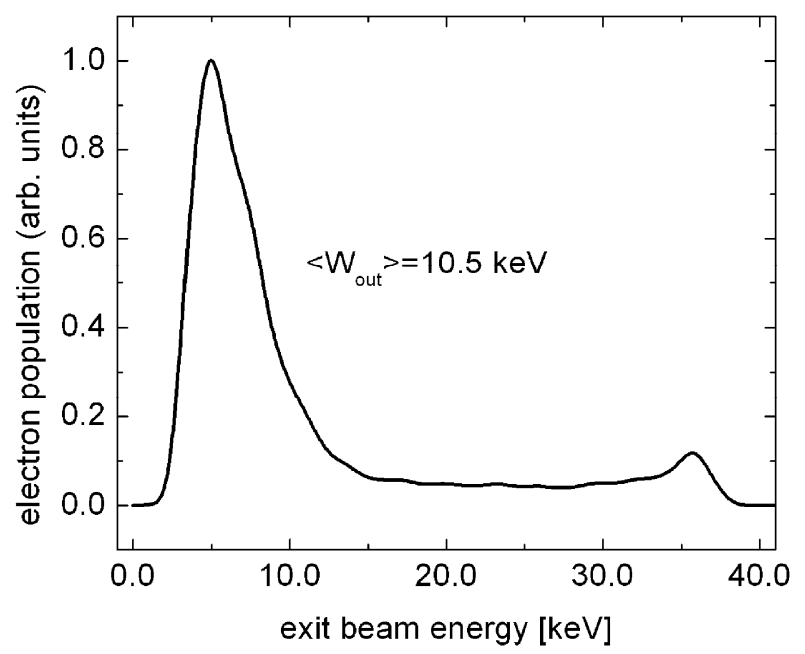

Figure 3. Kinetic-energy distribution for electrons collected at the end section $z=3.8 \mathrm{~cm}$ in the monotron configuration in Fig. 2.

\section{Conclusions}

One-dimensional monotron analysis incorporating a stepped electric-field profile indicates a maximum conversion efficiency of $57.0 \%$, almost a three-fold increase relative to the classical monotron with a uniform axial field. This prediction has been verified on the basis of two-cavity device designed to operate at $4.0 \mathrm{GHz}$ when excited by a $20 \mathrm{keV}$ electron beam. Upon applying a 1.0$\mathrm{kG}$ magnetic field, the simulation efficiency reaches $47.5 \%$, yet lower than the 1-D limit. This discrepancy is partially accounted for space-charge effects and radial variation of the electron velocity, not included in the analysis.

\section{References}

1. J. J. Müller and E. Rostas, "Un générateur à temps de transit utilisant un seul résonateur de volume", Helvet. Phys. Acta, vol. 13, no. 3, pp. 435-450, 1940.

2. Y. K. Yulpatov, "The excitation of oscillations in a cavity resonator by means of a relativistic electron beam", Radiophys. Quantum Electron., vol. 13, pp. 1374-1378, 1970.

3. J. J. Barroso, "Design facts in the axial monotron", IEEE Trans. Plasma Sci., vol. 28, pp. 652-656, June 2000.

4. J. J. Barroso, "A triple-beam $6.7 \mathrm{GHz}, 340 \mathrm{~kW}$ monotron", IEEE Trans. Electron Devices, vol. 48, pp. 815-817, April 2001.

5. V. P. Tarakanov, User's Manual for Code KARAT, Springfield, VA: Berkeley Research Associates Inc., 1994. 